\title{
The infrared supernova rate in starburst galaxies ${ }^{\star}$
}

\author{
F. Mannucci ${ }^{1}$, R. Maiolino ${ }^{2,3}$, G. Cresci ${ }^{4}$, M. Della Valle ${ }^{2}$, L. Vanzi ${ }^{5}$, F. Ghinassi ${ }^{6}$, V. D. Ivanov ${ }^{5}$, \\ N. M. Nagar ${ }^{2}$, and A. Alonso-Herrero ${ }^{7}$
}

\author{
1 Istituto di Radioastronomia, sezione di Firenze, Largo E. Fermi 5, 50125, Firenze, Italy \\ e-mail: filippo@arcetri.astro.it \\ 2 INAF, Osservatorio Astrofisico di Arcetri, Largo E. Fermi 5, 50125, Firenze, Italy \\ 3 Dipartimento di Astronomia, Univesità di Roma, via della vasca navale 86, Roma, Italy \\ ${ }^{4}$ Dipartimento di astronomia, Università di Firenze, Largo E. Fermi 5, 50125, Firenze, Italy \\ 5 ESO, Ave. Alonso de Cordova 3107, Casilla 19, Santiago 19001, Chile \\ 6 Telescopio Nazionale Galileo, Aptdo de Correos 565, 38700 Santa Cruz de la Palma, Canary Island, Spain \\ 7 Steward Observatory, Univ. of Arizona, Tucson, AZ85721, USA
}

Received 13 November 2002 / Accepted 13 February 2003

\begin{abstract}
We report the results of our ongoing search for extincted supernovae ( $\mathrm{SNe}$ ) at near-infrared wavelengths. We have monitored at $2.2 \mu \mathrm{m}$ a sample of 46 Luminous Infrared Galaxies and detected $4 \mathrm{SNe}$. The number of detections is still small but sufficient to provide the first estimate of supernova rate at near-infrared wavelengths. We measure a $\mathrm{SN}$ rate of $\mathrm{SN}_{\mathrm{r}}^{\mathrm{NIR}}=$ 7.6 $\pm 3.8 \mathrm{SNu}$ which is an order of magnitude larger than observed in quiescent galaxies. On the other hand, the observed nearinfrared rate is still a factor 3-10 smaller than that estimated from the far-infrared luminosity of the galaxies. Among various possibilities, the most likely scenario is that dust extinction is so high $\left(A_{V}>30\right)$ to obscure most SNe even in the near-IR.

The role of type Ia SNe is also discussed within this context. We derive the type Ia SN rate as a function of the stellar mass of the galaxy and find a sharp increase toward galaxies with higher activity of star formation. This suggests that a significant fraction of type Ia $\mathrm{SNe}$ are associated with young stellar populations.

Finally, as a by-product, we give the average $K$-band light curve of core-collapse $\mathrm{SNe}$ based on all the existing data, and review the relation between $\mathrm{SN}$ rate and far-infrared luminosity.
\end{abstract}

Key words. supernovae: general - supernovae: individual: SN 1999gd - supernovae: individual: SN 2000bg - infrared: stars

\section{Introduction}

The first report by Maiolino et al. (2002) on a sample of SNe detected in the near-infrared (NIR) has opened a new window for the search of these events. The presence of dust extinction can seriously reduce the measured SN rate in most of the galaxies, especially in starburst systems where dust obscuration is usually larger than in normal galaxies. This explains the puzzling result by Richmond et al. (1998) who found starburst and quiescent galaxies to have the same $\mathrm{SN}$ rate. A similar result was obtained by Navasardyan et al. (2001) who looked for $\mathrm{SNe}$ in interacting galaxies, finding no evidence for correlations between $\mathrm{SN}$ rate and galaxy properties.

The effects of dust extinction can be vastly reduced by observing in the NIR: at $2 \mu \mathrm{m}$ the extinction is about a factor of ten lower than in the optical $V$ band, allowing for a

Send offprint requests to: F. Mannucci, e-mail: filippo@arcetri.astro.it

* Based on observations collected at the European Southern Observatory, Chile (proposal 66.B-0417), at the Italian Telescopio Nazionale Galileo (TNG) operated on the island of La Palma by the Centro Galileo Galilei of the INAF (Istituto Nazionale di Astrofisica), and at the Steward Observatory 61" telescope. much deeper penetration into the dusty star-forming regions. This strategy was originally proposed by van Buren \& Norman (1989). The first monitoring campaign was conducted a decade ago and produced the detection of one event, SN 1994bu (van Buren et al. 1994). More recently, Grossan et al. (1999) monitored a large number of galaxies over two years but failed to detect extinguished $\mathrm{SNe}$, probably because of their poor spatial resolution (about $3^{\prime \prime}$ ). Bregman et al. (2000) used ISO/ISOCAM to look for mid-infrared spectral signatures of recent $\mathrm{SNe}$ in the nuclei of 10 nearby galaxies. However they failed to detect any emission from $\mathrm{SNe}$, such as the [NiII] emission line at $6.63 \mu \mathrm{m}$ which is very bright in SN 1987A (e.g., Arnett et al. 1989). This is probably due to the fact that the number of events expected from the far-IR flux (see Sect. 9) is lower than 0.5. Lorionov et al. (2002) reported the detection, while monitoring another event, of SN 2002cv, a bright type Ia SN showing a high extinction (Meikle \& Mattila 2002). A possible SN was also discovered by Mattila et al. (2002) in an archive UKIRT $K$-band image.

Most $\mathrm{SNe}$ in starburst galaxies are expected to be corecollapse events, i.e., to have massive progenitors. The role of type Ia SNe in these galaxies is the subject of an active debate. The progenitors of type Ia $\mathrm{SNe}$ are commonly identified with 
old white dwarfs in binary systems, but their rate could be enhanced in galaxies with active star formation (see, for example, Della Valle \& Livio 1994, and Della Valle \& Panagia 2003), as discussed in Sect. 8.

Near-infrared monitoring is needed not only to compare the SN production in quiescent and starburst galaxies, but also to obtain a complete estimate of the local SN rate to be compared with the rates at high redshift (Madau et al. 1998; Dahlén \& Fransson 1999; Sullivan et al. 2000). Finally, near-infrared searches are needed to detect a significant number of SNe in starburst galaxies; these events are expected to have peculiar properties: the circumstellar medium around them is likely to be very dense because they occur in molecular clouds and the mass-loss rates of the progenitor stars are expected to be higher because of the increased metallicity (Vink et al. 2001). The effects of this peculiar environment can be studied only when a significant number of events are discovered (Bressan et al. 2002).

In 1999 we started a program aimed at monitoring a sample of starburst galaxies, as discussed in Sect. 2. The most important differences with respect to previous monitoring programs are the use of 4-m class telescopes with higher spatial resolution, providing a deeper limiting magnitude especially for point sources, and the selection of galaxies with higher star-formation rate in order to have larger expected rates. This monitoring yielded the detection of $4 \mathrm{SNe}, 2$ of which were discovered by our group, SN 2001db (Maiolino et al. 2001) and SN 1999gw (Cresci et al. 2002). SN 2001db is the first SN detected in the NIR which has received a spectroscopic confirmation.

\section{The galaxy sample}

The $\mathrm{SN}$ rate $(\mathrm{SNr})$ is roughly proportional to the star-formation rate (SFR). The best way to have a galaxy sample selected in terms of SFR and not biased by extinction is to use the farinfrared (FIR) luminosity $L_{\text {FIR }}$ as measured with the IRAS 60 and $100 \mu \mathrm{m}$ bands. For all but the most dust-free galaxies this luminosity is closely proportional to the SFR as measured from the extinction-corrected optical lines and UV continuum and from the radio continuum (see, for example, Mannucci \& Beckwith 1995; Schaerer 1999; Cram et al. 1998).

Here we use a definition of FIR luminosity corresponding to the flux:

$$
f_{\mathrm{FIR}}=1.26 \times 10^{-14} C\left(2.58 \cdot f_{60}+f_{100}\right) \quad\left(\mathrm{W} / \mathrm{m}^{2}\right)
$$

(Helou 1988), where $f_{60}$ and $f_{100}$ are the IRAS fluxes at 60 and $100 \mu \mathrm{m}$ in Jy and $C$ is a numerical constant which depends on the $f_{60} / f_{100}$ ratio, and assumes values between 1.4 and 1.8. For galaxies with dust temperatures between 20 and $80 \mathrm{~K}$ and emissivity between 0 and 2, Eq. (1) is expected to estimate the total flux between 40 and $120 \mu \mathrm{m}$ within $1 \%$.

We selected galaxies from the various catalogs of IRAS galaxies (Sanders et al. 1995; Soifer et al. 1987; Kim \& Sanders 1998) having $L_{\text {FIR }}>10^{11.1} L_{\odot}$ which corresponds to $S N$ rates larger than about $0.4 \mathrm{SN} /$ year (see Sect. 9). We chose a limiting distance modulus of 36.5 , corresponding to about $200 \mathrm{Mpc}$, in order to have a good detection (signal-to-noise ratio of about 10) of an average SN (as SN 1980K having $M_{K}=-18.8$ at peak) with no extinction, given a typical limiting magnitude of $K \sim 18.5$. Three galaxies with distance modulus up to 37.3 but with very high FIR luminosities were also added to the sample. In conclusion, half of the sample is at distances below $100 \mathrm{Mpc}$. At $200 \mathrm{Mpc}$ the spatial scale is $200 \mathrm{pc} / \mathrm{arcsec}$, allowing for an easy detection of a point source even within the central kpc of the galaxies.

About a third of the galaxies having a spectroscopic classification suggesting the presence of a Seyfert nucleus, but in all cases the far-infrared luminosity seems to be dominated by star formation (see, for example, Corbett et al. 2002, and Thean et al. 2001). We excluded from the sample a few galaxies with low luminosity and large distance, and Mkn 231 as it contains an AGN whose luminosity outshines the rest of the galaxy.

Table 1 lists the 46 observed galaxies together with coordinates, absolute $B$ band magnitude, FIR luminosity, redshift and distance modulus.

\section{Observations}

The galaxies in Table 1 were observed between October 1999 and October 2001 using three different telescopes: the Telescopio Nazionale Galileo (TNG) equipped with the NIR camera ARNICA (Lisi et al. 1993) until September 2001 and NICS (Baffa et al. 2001) afterward, the ESO NTT telescope with SOFI (Lidman et al. 2000) and the Kuiper/Steward 61" infrared telescope (AZ61) on Mount Bigelow, equipped with a near-IR NICMOS3 camera.

In all cases we used the local realization of the " $K$ short" filter $\left(K^{\prime}\right)$, similar to $K$ but excluding the thermal part of the spectrum above $2.3 \mu \mathrm{m}$.

The sampling time was chosen in order to maximize the number of detections. At the limiting distance of our sample, $200 \mathrm{Mpc}$, an average SN remains above the detection limit for about 40 days, increasing to 110 days at $100 \mathrm{Mpc}$. To be conservative and concentrate the observations around nights with a full moon, we chose to observe the sample every 30 days. The final sampling is not very uniform as the atmospheric conditions and the actual scheduling on the three telescopes introduced some distortions on the original plan. We collected 234 observations, with an average number of 5.1 observations per galaxy (see Table 7).

The typical on-source integration times were about 20-30 min for NTT and TNG and 45-60 min for AZ61. Typical values of the seeing FWHM were 1.2 arcsec at TNG, 1.0 arcsec at NTT and $1.5 \operatorname{arcsec}$ at AZ61. The photometric calibration was achieved by using the Hunt et al. (1998) and Persson et al. (1998) standard stars.

\section{SN detection method, limiting magnitudes, and the $\mathrm{SNe}$ detections}

The comparison of the images of the same galaxies was performed with ISIS, a tool developed by Alard \& Lupton (1998), refined by Alard (2000) and available at http://www.iap.fr/users/alard/package.html. First, all the images of a galaxy were aligned by using field stars 
Table 1. The galaxy sample. The name, coordinates, absolute $B$-band magnitude, $\log \left(L_{\mathrm{FIR}} / L_{\odot}\right)$, and distance modulus for $H_{0}=70 \mathrm{~km} \mathrm{~s}^{-1} \mathrm{Mpc}^{-1}$.

\begin{tabular}{|c|c|c|c|c|c|c|}
\hline Galaxy & \multicolumn{2}{|c|}{ RA (J2000) Dec. } & $M_{B}$ & 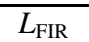 & $z$ & $(m-M)$ \\
\hline NGC 34 & 001106.5 & -120626 & -19.42 & 11.34 & 0.020 & 34.46 \\
\hline NGC 232 & 004245.6 & -233339 & -20.44 & 11.23 & 0.022 & 34.70 \\
\hline $\mathrm{MCG}+12-02-001$ & 005403.6 & +730512 & -21.05 & 11.29 & 0.016 & 34.03 \\
\hline IC 1623 & 010747.2 & -173025 & -20.56 & 11.38 & 0.019 & 34.50 \\
\hline UGC 2369 & 025401.4 & +145814 & -21.17 & 11.44 & 0.031 & 35.49 \\
\hline IRAS $03359+1523$ & 033846.9 & +153255 & -20.30 & 11.38 & 0.035 & 35.76 \\
\hline MCG -03-12-002 & 042120.0 & -184845 & -21.09 & 11.30 & 0.032 & 35.51 \\
\hline NGC 1572 & 042242.7 & -403602 & -21.51 & 11.16 & 0.020 & 34.46 \\
\hline NGC 1614 & 043359.8 & -083444 & -21.19 & 11.41 & 0.016 & 33.96 \\
\hline IRAS 05189-2524 & 052101.4 & -252145 & -20.59 & 11.89 & 0.042 & 36.11 \\
\hline ESO 255-IG007 & 062723.1 & -471044 & -16.88 & 11.67 & 0.039 & 35.89 \\
\hline NGC 2623 & 083824.0 & +254517 & -21.20 & 11.47 & 0.018 & 34.37 \\
\hline IRAS $08572+3915$ & 090025.4 & +390354 & - & 11.99 & 0.058 & 36.85 \\
\hline UGC 4881 & 091555.5 & +441949 & -20.90 & 11.57 & 0.040 & 36.01 \\
\hline UGC 5101 & 093551.4 & +612111 & -20.78 & 11.90 & 0.039 & 36.00 \\
\hline $\mathrm{MCG}+08-18-012$ & 093630.7 & +482810 & -20.16 & 11.19 & 0.026 & 35.07 \\
\hline IC 563/IC 564 & 094620.3 & +030243 & -21.58 & 11.10 & 0.020 & 34.50 \\
\hline NGC 3110 & 100401.9 & -062829 & -21.77 & 11.10 & 0.016 & 34.12 \\
\hline IC 2545 & 100604.5 & -335303 & -20.78 & 11.57 & 0.034 & 35.64 \\
\hline IRAS $10173+0828$ & 102000.0 & +08 1335 & -19.11 & 11.68 & 0.048 & 36.42 \\
\hline NGC 3256 & 102551.8 & -435409 & -21.57 & 11.44 & 0.009 & 32.67 \\
\hline IRAS $10565+2448$ & 105918.1 & +243234 & -21.76 & 11.87 & 0.042 & 36.20 \\
\hline Arp 148 & 110353.9 & +405100 & - & 11.50 & 0.034 & 35.76 \\
\hline MCG +00-29-023 & 112112.2 & -025903 & -20.14 & 11.36 & 0.024 & 34.98 \\
\hline IC 2810/UGC 6436 & 112545.0 & +144036 & -20.12 & 11.50 & 0.034 & 35.69 \\
\hline NGC 3690 & 112831.9 & +583345 & -22.04 & 11.72 & 0.011 & 33.38 \\
\hline IRAS $12112+0305$ & 121345.7 & +024839 & -20.21 & 12.19 & 0.072 & 37.32 \\
\hline ESO 507-G070 & 130252.1 & -235519 & -21.28 & 11.31 & 0.021 & 34.63 \\
\hline UGC 8335 & 131529.2 & +620711 & -20.22 & 11.60 & 0.031 & 35.51 \\
\hline UGC 8387 & 132035.3 & +340822 & -20.38 & 11.52 & 0.023 & 34.91 \\
\hline NGC 5256 & 133817.7 & +481634 & -21.80 & 11.37 & 0.027 & 35.31 \\
\hline NGC 5257/5258 & 133953.1 & +005022 & -21.83 & 11.37 & 0.022 & 34.80 \\
\hline Mk 273 & 134442.1 & +55 5313 & -20.22 & 12.10 & 0.038 & 35.92 \\
\hline NGC 5331 & 135216.5 & +020609 & -20.72 & 11.43 & 0.033 & 35.62 \\
\hline Arp 302 & 145700.3 & +243656 & -20.84 & 11.59 & 0.034 & 35.67 \\
\hline Mk 848 & 151806.3 & +424437 & -20.41 & 11.72 & 0.040 & 36.07 \\
\hline IRAS $15250+3609$ & 152659.4 & +355838 & -21.21 & 11.89 & 0.053 & 36.74 \\
\hline Arp 220 & 153457.3 & +233012 & -20.78 & 12.12 & 0.018 & 34.37 \\
\hline NGC 6090 & 161140.3 & +522726 & -21.51 & 11.35 & 0.029 & 35.42 \\
\hline IRAS 16164-0746 & 161911.8 & -075403 & -20.51 & 11.40 & 0.027 & 34.93 \\
\hline NGC 6240 & 165258.9 & +022403 & -21.68 & 11.85 & 0.024 & 34.96 \\
\hline IRAS 17208-0014 & 172321.9 & -001700 & -20.13 & 12.30 & 0.043 & 36.18 \\
\hline IC $4687 / 86$ & 181339.6 & -574331 & -20.60 & 11.35 & 0.017 & 34.12 \\
\hline IRAS 18293-3413 & 183240.2 & -341126 & -19.50 & 11.63 & 0.018 & 34.28 \\
\hline NGC 6926 & 203306.2 & -020140 & -22.15 & 11.11 & 0.020 & 34.52 \\
\hline NGC 7130 & 214819.5 & -345709 & -21.09 & 11.21 & 0.016 & 33.96 \\
\hline
\end{tabular}

or the galaxy nucleus. Second, the image with the best pointspread-function (PSF) was identified and used as reference for the comparison with the other images. In order to minimize the residuals without losing spatial resolution, the PSF of the reference image was convolved with an appropriate kernel determined, for each image pair, by a linear fit. ISIS was found to give better results than other similar tasks, such as the IRAF task "immatch". Finally, the images were normalized to the same total flux and subtracted.

On average ISIS is able to subtract $97 \%$ of the total galaxy flux, with a mode of $98 \%$. The residuals were usually concentrated at the position of the galactic nuclei, where the emission has a strong radial gradient. Non circular structures such as the diffraction spikes on the spider arms are not reproduced by the ISIS kernel. The presence of these residuals makes the detection of nuclear $\mathrm{SNe}$ much more difficult, as discussed below.

Outside the nuclear regions, i.e., about 1 arcsec away from the galaxy center, the SN detection limit was estimated through simulations by adding point sources to the subtracted image and trying to recover them. The detection was performed by using SExtractor (Bertin \& Arnouts 1996) with parameters set 
Table 2. SN detection limits.

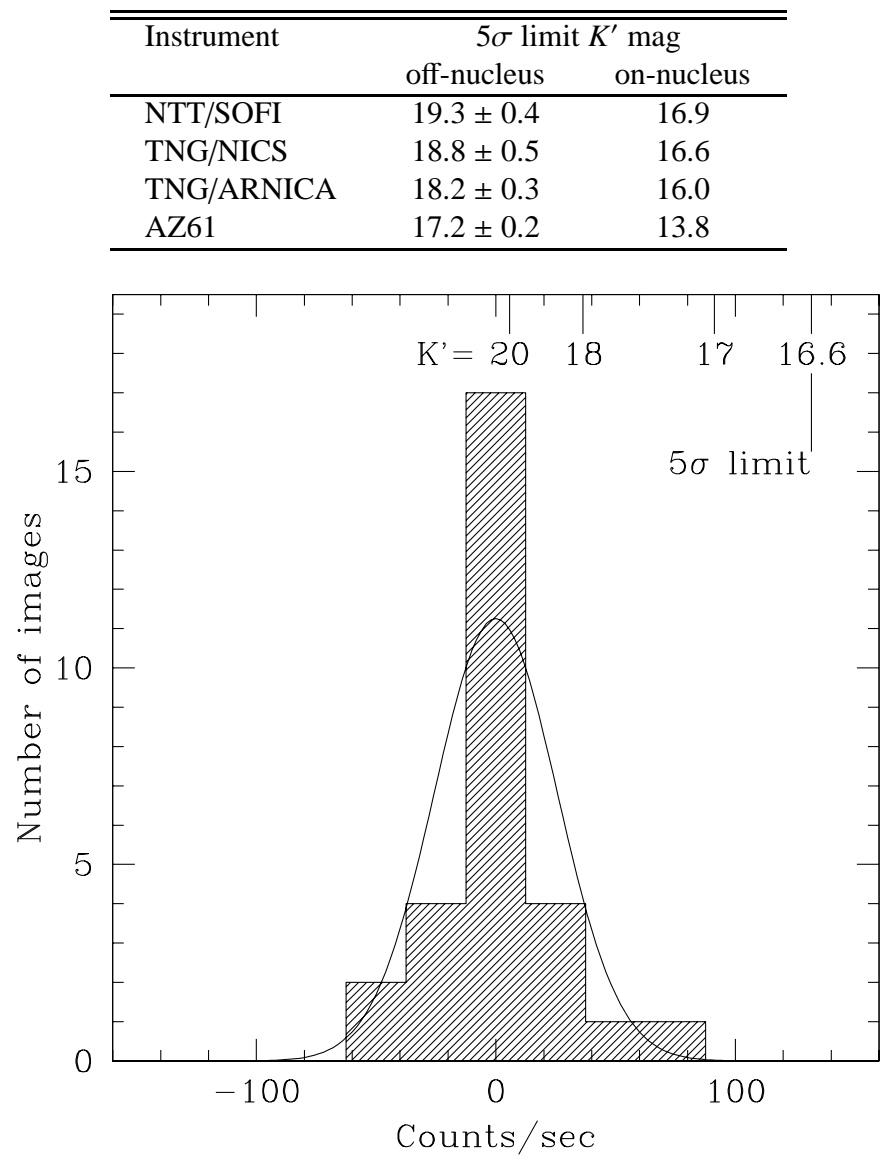

Fig. 1. Histogram of the nuclear residuals in the TNG/NICS images. The residuals are plotted in counts/sec and the scale near the top shows the corresponding magnitudes. The Gaussian fit and the $5 \sigma$ limit of $K^{\prime}=16.6$ are also shown

to have secure detections of point sources $(2.5 \sigma$ per pixel over an area of 6 pixels, corresponding to about a $6 \sigma$ detection). The limiting magnitudes, defined at a completeness level of $50 \%$, are listed in Table 2 for each telescope, together with their spread because of variable seeing, transparency and integration time. These values are in good agreement (within $0.1 \mathrm{mag}$ ) with the expectation from the parameters of the detector and of the exposures.

In the central arcsec, the presence of the PSF subtraction residuals makes it possible to detect only brighter $\mathrm{SNe}$. To estimate this limit we measured the residuals inside a 1.5 arcsec aperture on each subtracted image, as shown in Fig 1. The magnitude corresponding to $5 \sigma$ positive counts is taken as the detection limit. This is actually an upper limit to the real sensitivity as in doing this we assume than the measured residuals are instrumental and no $\mathrm{SNe}$ are actually present.

The analysis of the data revealed $4 \mathrm{SNe}$, listed in Table 3. Two objects, SN 1999gd and SN 2000bg, were independently detected by us but they had been already discovered by optical searches a few days before. Their $K$-band magnitudes are listed in Table 4. The other two SNe, discovered in our images, are presented in Maiolino et al. (2002). All the SNe were discovered out of the galactic nuclei; the event closest to the
Table 3. Detected SNe.

\begin{tabular}{cccccc}
\hline \hline SN & Type & Galaxy & \multicolumn{2}{c}{ Discovery } & ref. \\
& & & Date & Band & \\
\hline $1999 \mathrm{gd}$ & Ia & NGC 2623 & 1999 Nov. 24 & Opt. & 1,2 \\
$1999 \mathrm{gw}$ & II & UGC 4881 & 1999 Dec. 16 & $K^{\prime}$ & 3,4 \\
$2000 \mathrm{bg}$ & IIn & NGC 6240 & 2000 Apr. 01 & Opt. & 5,6 \\
$2001 \mathrm{db}$ & II & NGC 3256 & 2001 Jan. 09 & $K^{\prime}$ & 3,7 \\
\hline
\end{tabular}

References. 1: Li (1999); 2: Filippenko \& Garnavich (1999); 4: Cresci et al. (2002). 3: Maiolino et al. (2002); 5: Sato \& Li (2000); 6: Jha \& Brown (2000); 7: Maiolino et al. (2001);

Table 4. $K^{\prime}$ light curves of SN 1999gd and SN 2000bg.

\begin{tabular}{cc}
\hline \hline \multicolumn{2}{c}{ Date } \\
\hline \multicolumn{2}{c}{ SN 1999gd } \\
\hline 1999 Dec. 15 & $16.0 \pm 0.2$ \\
2000 Jan. 25 & $17.4 \pm 0.2$ \\
2000 Feb. 11 & $>18.0$ \\
\hline \multicolumn{2}{c}{ SN 2000bg } \\
\hline 2000 Mar. 14 & $>17.0$ \\
2000 Apr. 10 & $16.0 \pm 0.3$ \\
2000 May 12 & $16.0 \pm 0.3$ \\
\hline
\end{tabular}

nucleus of its host galaxy is SN $1999 \mathrm{gw}$ at $3.5^{\prime \prime}$ distance. As discussed in Maiolino et al. (2002), SN 2001db is highly extincted $\left(A_{V} \sim 5.6\right)$, while the available data do not allow to constrain the extinction of the other $\mathrm{SNe}$.

\section{Computing the infrared $\mathrm{SN}$ rate}

This paper is devoted to computing the near-IR SN rate in starburst galaxies by comparing the number of detections with the number of expected events. To do this we need a few ingredients which will be the subject of the following sections:

- The computation of the expected number of detections is based on the estimate of the control time, i.e., the amount of time that a $\mathrm{SN}$ in a given galaxy remains above the detection limit (as defined by Zwicky 1938). Therefore it is critical to have a good knowledge of the NIR light curve, as discussed in Sect. 6.

- The expected number of exploding core-collapse SNe is also related to the total SFR and therefore is expected to be proportional to the FIR luminosity of the target galaxies. This proportionality is discussed in Sect. 7.

- Type Ia SNe are usually assumed to have old progenitors and therefore to be more related to the total mass in stars of the galaxies than to the current or recent SFR. This might not be true for all types of Ia $\mathrm{SNe}$ as discussed by several authors. In Sect. 8 we discuss the relation between type Ia $\mathrm{SN}$ rate and stellar mass along the Hubble morphological types sequence. We will discuss the effect of including the type Ia $\mathrm{SNe}$ on the infrared $\mathrm{SN}$ rate.

- Very different detection limits apply to nuclei and to the rest of the galaxies; as a consequence, the $\mathrm{SN}$ rates depend of the fraction of starburst activity contained in the inner few arcsec of the galaxies. In Appendix A we discuss the existing FIR data, concluding that they do not really constrain 
the size of the starbursts, even if in many cases most of the star formation activity is concentrated in the nuclear region.

- Section 9 contains the actual computation of the SN rate. This is done considering three scenarios for the spatial dimension of the starbursts: we consider the two limiting cases that the fraction of starburst in the central 2 arcsec is (a) negligible, and (b) dominant, and an intermediate case in which $80 \%$ of the starburst is contained in the nucleus. The rates are also computed both by considering the corecollapse $\mathrm{SNe}$ only and also by including the type Ia SNe.

\section{The $K$-band light curve of the core-collapse SN}

The evolution of the $K$ band luminosity of the core-collapse SN is currently not very well constrained. Mattila \& Meikle (2001) made a compilation of the 12 curves available at that time in the $K$ band to derive the average light curve. The small number of available measures is due to the fact that NIR observations are not available for all the SNe and that only events with a well known epoch of the optical maximum are considered. They divided the core-collapse supernovae in two classes, the "ordinary events" showing decline rate similar to the optical band, and the "slow declining events" with NIR luminosity almost constant for hundreds of days after the optical maximum. We have added 9 new light curves for a total number of more than 200 NIR observations (see Table 5 and references therein). We have also included SN 1987A which, in the near-IR, has a less peculiar behavior than in the optical.

The resulting sample is not complete, so it should not be used for statistics. Nevertheless the fraction of the slow declining events appears to be not negligible: 7 out of $22 \mathrm{SNe}$ show a peculiar behavior at some point of their evolution, and 5 of them are classified as type IIn SNe from the optical spectra. The fraction is even higher, 7 out of 12 , if only the SN with observations after 4 months from the maximum are considered. Such a high fraction is certainly due to an observational bias, as SNe with NIR excess are more often observed at such late times (see, for example, Gerardy et al. 2002). SN 1987A shows a peak luminosity similar to the other events and a decline rate intermediate between the ordinary and the slow declining events.

This brightening or stabilization of the luminosity at NIR wavelengths is usually attributed to thermal emission from dust forming in the ejecta or present in the pre-existing circumstellar medium (e.g., Gerardy et al. 2002), whereas the heating could be due to the interaction between the ejecta and the circumstellar medium (Fassia et al. 2000, 2001; Di Carlo et al. 2002). For this reason the effect is expected to be more common in the events occurring in the dense star forming regions as the type IIn SNe (Schlegel 1990). These SNe become much brighter than the others, but their constant luminosity makes their detection by the usual "blinking" technique almost impossible after the maximum light.

The 15 "ordinary events" show an inhomogeneous behavior during the first 4 months. Most of them reach a maximum 10-20 days after the optical peak, and decline linearly afterward. The NIR luminosity peak of core-collapse SNe is similar to that of the type Ia, estimated to be $M_{K}=-18.825$ days after the optical maximum (Meikle 2000), even if the core-collapse SNe are usually much fainter in the optical. SN 1999em is the least luminous event, with $K$ magnitude about 1 mag fainter at the optical maximum than any other measured $\mathrm{SN}$, and shows a very delayed peak, occurring at about 70 days after the optical maximum. The luminosity spread among the $\mathrm{SNe}$ of each class is comparable with the spread among the different classes, i.e., there is no evidence of systemic differences for the corecollapse SNe of different sub-types.

After the first 120 days, "ordinary" SNe show similar decline rates of about $0.025 \mathrm{mag} / \mathrm{day}$, much faster than the optical decline rate of about $0.010 \mathrm{mag} / \mathrm{day}$ at comparable stages corresponding to the ${ }^{56} \mathrm{Co}$ decay (0.0098 mag/day, e.g., Cappellaro \& Turatto 2001).

The spread of the absolute magnitude near the $K$-band peak is about $3 \mathrm{mag}$, and about $1.5 \mathrm{mag}$ at later times. To account this large spread we have defined the upper and lower envelopes of the distributions (see Fig. 2 and Table 6) and will use these curves to compute the maximum and minimum $\mathrm{SN}$ rate expected from the SFR. The upper envelope is defined by SNe 1979C and 1982R, the lower envelope by SN 1999em. A few SNe, such as 1983I and 1993J, are fainter than 1999em at epochs between 50 and 120 days, but are brighter near the optical maximum and therefore were not considered for the lower envelope.

For the average light-curve we used SN 1980K, an event showing a linear decline and a peak magnitude near the average value. We preferred to use an observed event instead of an average between the two envelopes since the latter is strongly dependent on peculiar sub- or super-luminous events and does not correspond to any observed SN.

\section{SN rate and FIR luminosity}

To compute the number of expected SNe in our sample, we need to discuss the relation between FIR luminosity and $\mathrm{SN}$ rate and the role of type Ia $\mathrm{SNe}$. These will be the subject of the next two sections.

Several authors have computed the $\mathrm{SN}$ rates as a function of the host galaxy morphological type. These estimates are based on optical observations of local galaxies having dust contents and SFRs much lower than the objects in our sample.

Rates are generally normalized to the luminosity of the parent galaxy, either the $B$ luminosity or $L_{\mathrm{FIR}}$. The former normalization is applied when using the $\mathrm{SN}$ unit ( $\mathrm{SNu}$ ), i.e., the number of SNe per century per $10^{10} L_{\odot}$ of $B$ luminosity. This luminosity has an ambiguous physical meaning: in starburst galaxies it is roughly proportional to the SFR, in quiescent galaxies it is related to the total mass in stars, and in dusty starbursts it is very sensitive to the dust content and distribution. Here we will use the rate of $0.93 \pm 0.28 \mathrm{SNu}$ by Cappellaro et al. (1999, Table 4) for galaxies later than Sb and including the rates for types $\mathrm{II}$ and $\mathrm{Ib} / \mathrm{c}$.

For galaxies with a significant SFR it is more useful, for many applications and especially when the core-collapse $\mathrm{SNe}$ are considered, to normalize the observed number of SNe to the FIR luminosity, known to be proportional to the SFR. Several 


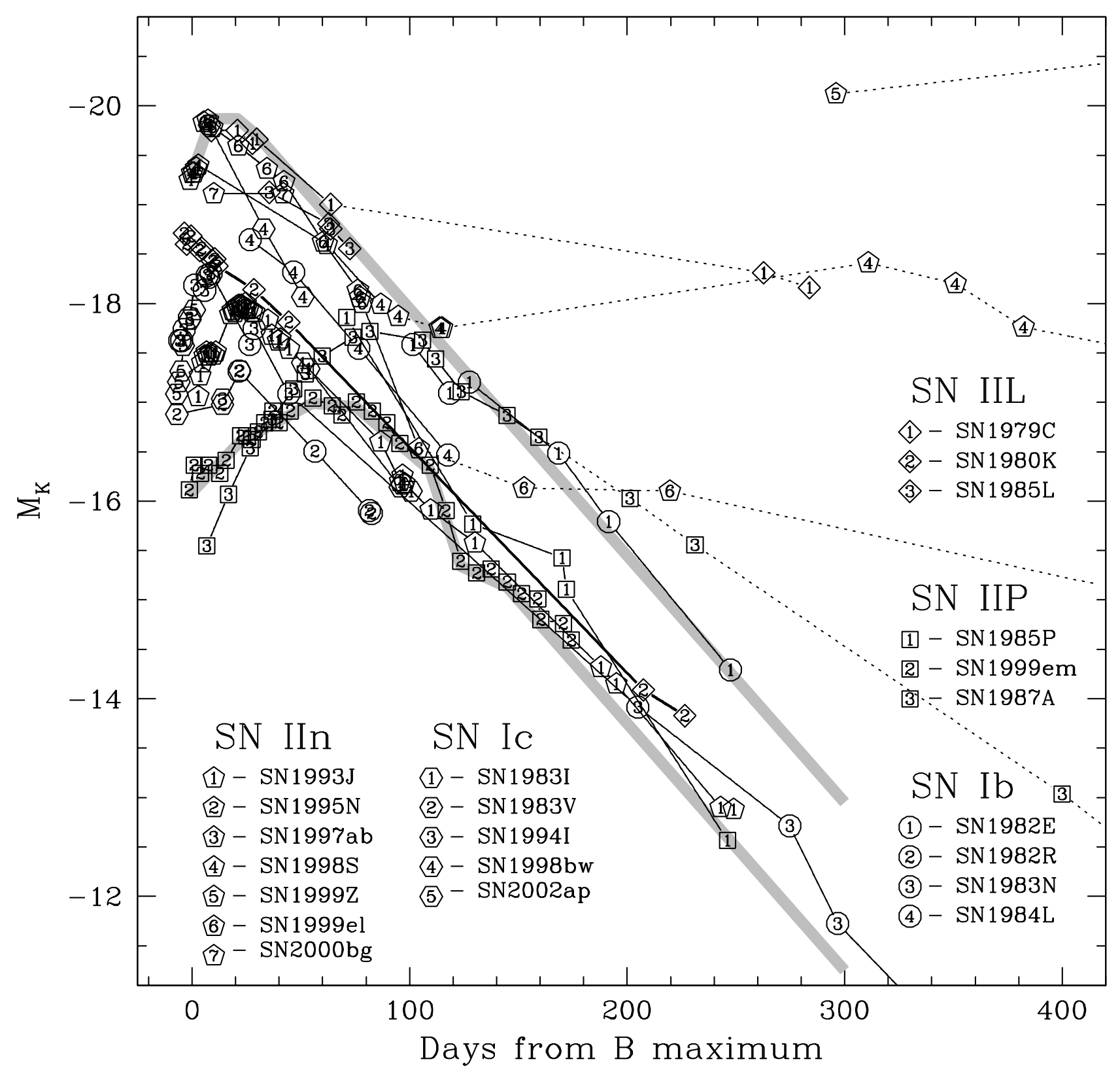

Fig. 2. $K$-band light-curves of all the core-collapse supernovae available in the literature. The upper- and lower-envelope used for the computation of the control time are shown by the thick grey lines. The thick solid line shows SN 1980K. The part of the curves connected with dotted lines are varying too slowly to produce a detection and therefore were not considered to compute the envelopes. Some objects lie outside of the plot because only observations at epoch $>400$ days are available.

indirect methods based on radio, FIR or mid-infrared observations have been used to derive the $\mathrm{SN}$ rate in obscured starburst galaxies, as described in van Buren \& Greenhouse (1994) and Mattila \& Meikle (2001). These authors have derived the relation between the $\mathrm{SN}$ rate and $L_{\mathrm{FIR}}$ using galaxies of low FIR luminosity, and therefore a large extrapolation is needed to apply these results to our sample. We have extended the observed range toward high luminosities by using the relation between radio luminosity and SN rate in Condon \& Yin (1990) and data in Hackenberg et al. (2000) and Wilson et al. (1991). The SN rate appears to be proportional to $L_{\mathrm{FIR}}$ with a small scatter for luminosities between 1.6 and $30 \times 10^{10} L_{\odot}$ as shown in Fig. 3, without the detection of any dependence on the lumi- nosity of the galaxy or on the SFR. Using the definition of $L_{\mathrm{FIR}}$ in Eq. (1), we obtain the relation:

$\mathrm{SNr}=(2.4 \pm 0.1) \times\left(L_{\mathrm{FIR}} / 10^{10} L_{\odot}\right) \mathrm{SN} / 100 \mathrm{yr}$

which, we stress, is based on indirect measures of the $\mathrm{SN}$ rate. A similar value of the proportionality factor (2.7) was also obtained by Mattila \& Meikle (2001) even though they use a slightly different definition of $L_{\mathrm{FIR}}$. In that case the data range was below $L_{\mathrm{FIR}}=10^{10.8} L_{\odot}$, a factor of two below the faintest galaxy in our sample. These authors discuss how this factor is in good agreement with the expectations from the models of galaxy stellar populations which predict the FIR luminosity from the UV flux and the SN rate from the product of the 
Table 5. Core-collapse SNe used to define the NIR light curve. Name, type, epoch of optical maximum, distance modulus, number of $K$ band observations and references are reported.

\begin{tabular}{llcccl}
\hline \hline SN & Type & $\begin{array}{c}\text { Epoch } \\
\text { (JD) }\end{array}$ & $(m-M)$ & $N_{\text {obs }}$ & ref. \\
\hline 1979 C & IIL & 2443979 & 31.03 & 8 & 1 \\
$1980 \mathrm{~K}$ & IIL & 2444543 & 28.78 & 13 & 2 \\
$1982 \mathrm{E}$ & Ib & 2445057 & 31.39 & 7 & 3 \\
$1982 \mathrm{R}$ & Ib & 2445248 & 31.22 & 5 & 3,4 \\
$1983 \mathrm{I}$ & Ic & 2445443 & 31.30 & 6 & 5 \\
$1983 \mathrm{~N}$ & Ib & 2445533 & 28.49 & 17 & 1,5 \\
$1983 \mathrm{~V}$ & Ic & 2445672 & 31.30 & 4 & 6 \\
$1984 \mathrm{~L}$ & Ib & 2445943 & 31.52 & 6 & 5 \\
$1985 \mathrm{~L}$ & IIL & 2446227 & 31.51 & 5 & 1 \\
$1985 \mathrm{P}$ & IIP & 2446349 & 30.47 & 6 & 1 \\
$1987 \mathrm{~A}$ & IIP & 2446849 & 18.55 & many & 7 \\
$1993 \mathrm{~J}$ & IIn & 2449095 & 27.80 & 25 & $8-14$ \\
$1994 \mathrm{I}$ & Ic & 2449451 & 29.60 & 2 & 15 \\
$1995 \mathrm{~N}$ & IIn & 2449539 & 32.07 & 14 & 16 \\
$1997 \mathrm{ab}$ & IIn & 2450186 & 33.49 & 3 & 16 \\
$1998 \mathrm{~S}$ & IIn & 2450890 & 31.30 & 17 & $1,16,17$ \\
$1998 \mathrm{bw}$ & Ic & 2450944 & 32.96 & 4 & 18 \\
$1999 \mathrm{Z}$ & IIn & 2451219 & 36.61 & 3 & 16 \\
$1999 \mathrm{el}$ & IIn & 2451572 & 32.53 & 14 & 16,19 \\
$2000 \mathrm{bg}$ & IIn & 2451636 & 35.11 & 2 & 20 \\
$1999 \mathrm{em}$ & IIP & 2451483 & 29.38 & 35 & 21 \\
2002ap & Ic & 2453314 & 29.52 & 6 & $22-25$ \\
\hline
\end{tabular}

References. 1: Mattila \& Meikle (2001); 2: Dwek et al. (1983); 3: Graham (1985); 4: Muller (1982); 5: Elias et al. (1985); 6: Clocchiatti et al. (1997); 7: Bouchet et al. (1989); 8: Calamai et al. (1993); 9: Odewahn et al. (1993); 10: Lawrence et al. (1993); 11: Romanishin (1993); 12: Smith (1993); 13: Kidger et al. (1993); 14: Matthews et al. (2002); 15: Grossan et al. (1999); 16: Gerardy et al. (2002); 17: Fassia et al. (2000); 18: Patat et al. (2001); 19: Di Carlo et al. (2002); 20: this paper; 21: Hamuy et al. (2001); 22: Motohara et al. (2002); 23: Yoshii et al. (2002); 24: Mattila et al. (2002) 25: Mannucci et al., in prep.

Table 6. Reference $K$-band light curve and lower- and upperenvelopes for core-collapse SNe. Epoch refers to the optical maximum.

\begin{tabular}{rccc}
\hline \hline Epoch & Lower & SN 1980K & Upper \\
\hline-1 & -16.07 & -18.66 & -19.25 \\
9 & -16.30 & -18.44 & -19.87 \\
22 & -16.60 & -18.23 & -19.85 \\
30 & -16.70 & -18.11 & -19.70 \\
55 & -16.98 & -17.57 & -19.08 \\
75 & -16.95 & -17.11 & -18.58 \\
109 & -16.32 & -16.34 & -17.73 \\
123 & -15.37 & -16.02 & -17.38 \\
144 & -15.14 & -15.54 & -16.85 \\
300 & -11.25 & -11.64 & -12.95 \\
\hline
\end{tabular}

SFR times the integral of the Initial Mass Function (IMF) over the appropriate mass range. Finally, Cappellaro et al. (1999) obtained the ratio between the rate of unobscured $\mathrm{SNe}$ from optical monitoring and the FIR luminosity of a large number of galaxies from the RC3 catalog. They obtained a coefficient of 2.5, in good agreement with our calibration.

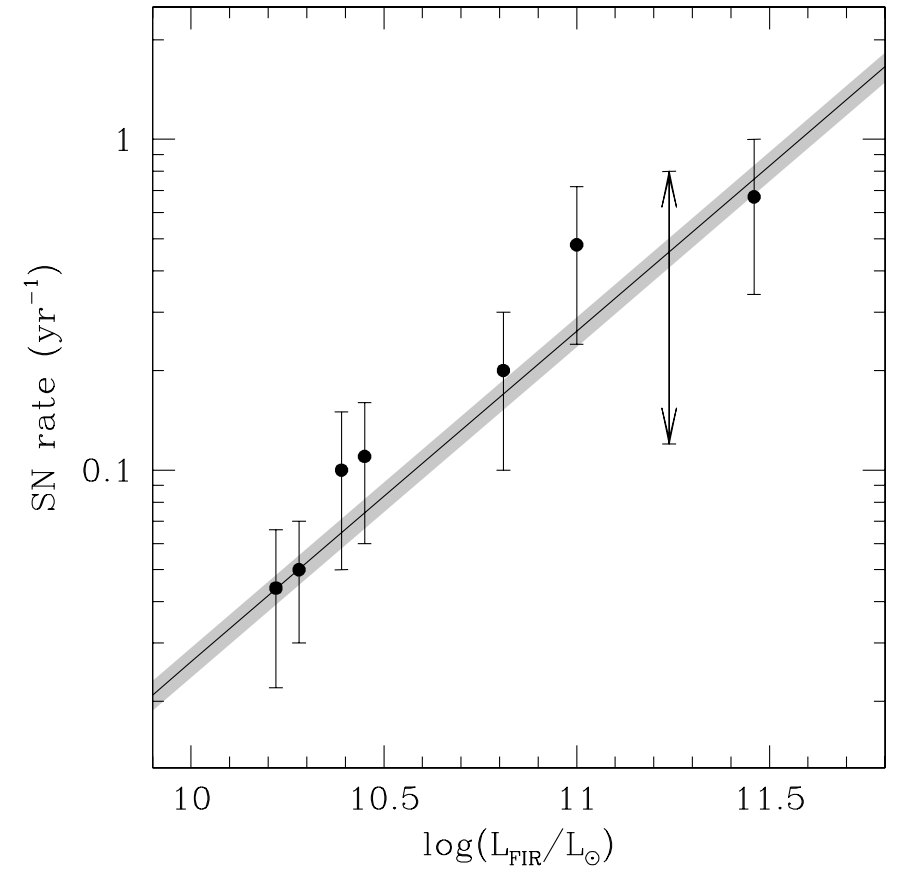

Fig. 3. SN rates vs. $L_{\mathrm{FIR}}$ for a sample of galaxies with active star formation. From left to right: UGC 1347 (Hackenberg et al. 2000); NGC 253 (Mattila \& Meikle 2001 and references therein); NGC 7673 (Condon \& Yin 1990) M 82 (Mattila \& Meikle 2001 and references therein); NGC 4038 (Neff \& Ulvestad 2000); NGC 1068 and NGC 7469 (Wilson et al. 1991). When not available, errors of $50 \%$ of the measured values were assumed. The solid line shows a fit to these points passing through the origin, the shaded region the associated $1 \sigma$ error. The double arrow shows the upper- and lower limits for NGC 7771 (Smith et al. 1999) due to a poorly known correction for incompleteness.

Smith et al. (1999) measured the SN rate in the central 10" of NGC 7771, a starburst galaxy with $\log \left(L_{\mathrm{FIR}} / L_{\odot}\right)=11.25$. This value cannot be used for the above relation because it only referred to the central part of the galaxy. To correct this SN rate to a value appropriate to the total galaxy, we assume that the FIR luminosity is well traced by the $15 \mu \mathrm{m}$ emission (see, for example, Elbaz et al. 2002) and use the growth profile by Dale et al. (2000). This is probably an overcorrection, as the size of the region emitting $80 \%$ of the $15 \mu$ m luminosity is 54 " while Zink et al. (2000) obtain an upper limit to the $100 \mu \mathrm{m}$ emitting region of about $20^{\prime \prime}$. In Fig. 3 we show the upper- and lower-limits to the SN rate in NGC 7771: the value predicted by Eq. (4) is within the permitted range.

\section{The role of type la SNe}

The progenitors of the type Ia $\mathrm{SNe}$ are expected to be old systems containing a white dwarf accreting mass from a companion star. If all Ia $\mathrm{SNe}$ are produced by the same old progenitors, their properties (as absolute magnitude at maximum, decline rate and expansion velocity of the ejecta) should not correlate with the current SFR of their host location. On the other hand several authors have discovered systematic differences between type Ia $\mathrm{SNe}$ in galaxies of different Hubble types in terms of optical spectral properties (Branch \& van den Berg 1993, 


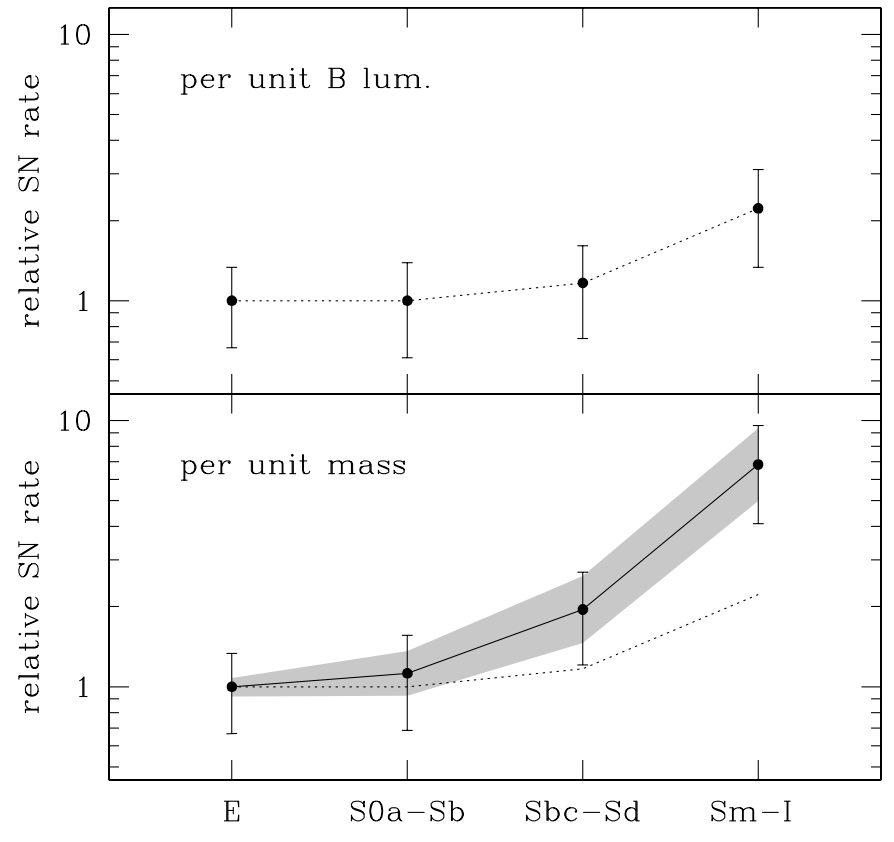

Fig. 4. Upper panel: type Ia SN rate by Cappellaro et al. (1999) normalized to that in the elliptical galaxies. The rates were measured in $\mathrm{SNu}$, i.e., were compared to the $B$ luminosity of the galaxies. The bars show the errors in the observed rates. Lower panel: the relative SN rate are normalized to the total stellar mass of the galaxies as in Bell \& de Jong (2001). A factor of 7 of increase is observed from the ellipticals to the irregular galaxies. The grey region show the effect of the uncertainties on $\mathrm{M} / \mathrm{L}$ due to the color spread in each galaxy Hubble type. The dotted line is the same of the upper panel and is shown as reference.

and references therein), photometric differences (Della Valle \& Panagia 1992), photometric evolution (Hamuy et al. 2000) and rates of occurrence (Della Valle \& Livio 1994).

If the progenitors are old, the number of type Ia SNe should also roughly correlate with the total stellar mass. This fact is barely detectable from the $\mathrm{SN}$ rate expressed in $\mathrm{SNu}$ as the $B$ luminosity is not a good tracer of stellar mass. On the contrary, the luminosity in the $K$ band is a much better tracer, as discussed for example by Brinchmann \& Ellis (2000). The dominating uncertainties are due to the age of the stellar population, and Bell \& de Jong (2001) propose a method to correct for this effect from the observed colors. We have therefore corrected the rates in Cappellaro et al. (1999) by using the average $B-K$ colors for galaxies of the various Hubble type by Fioc $\&$ Rocca-Volmerange (1999) and the corrections to the $\mathrm{M} / \mathrm{L}$ ratio by Bell \& de Jong (2001). show an increase of about a factor of 2 of the SN rate in the Sbc-Sd galaxies over that in the ellipticals, rising to about a factor of 7 for Sm-I galaxies. These values could be affected by systematic errors, but the results are consistent with the hypothesis of a substantial enhancement of the type Ia SN rate due to young stars. The details of this computation and the the relation between the stellar mass and the type Ia rate will be subject of a forthcoming paper.

Based on this kind of evidences, Della Valle \& Livio (1994) found that late type spirals are more prolific producers of type Ia SNe and proposed the association of part of the type Ia SNe with younger stellar populations.
For these reasons, we will make the calculations under two opposite limiting hypothesis that (1) none, and (2) all the type Ia SNe derive from young progenitors. In the second hypothesis the expected SN rate is raised by about $30 \%$ as this is the fraction of type Ia SNe vs. the core-collapse SN measured by Cappellaro et al. (1999) for galaxies later than Sbc. In this case the number of detected SN is also increased, as the type Ia SN 1999gd must be included in the sample, and the two effects (larger expected rates and more observed events) tend to cancel each other.

\section{The infrared $\mathrm{SN}$ rate}

The rates we can finally compute depend on the control time and therefore on the limiting magnitude. In the central 1 or 2 arcsecs of the galaxy nucleus the capability of detecting $\mathrm{SNe}$ is dimmed because of the presence of residuals after the subtraction, as discussed in Sect. 4. Although the area affected by the residuals is a small fraction of the total galaxy, this part could contain a significant fraction of events because it could host a compact nuclear starburst region at the bottom of the potential well. In Appendix A we discuss the information on the size of the starburst region coming from the mid-IR and far-IR data, and we conclude that many galaxies might be dominated by a compact starburst but that the spatial resolution of the data is too poor to obtain definitive conclusions.

As a consequence we compute the rates in the two limiting cases that the FIR flux in the nuclear region is (a) negligible (labeled as off-nuclear case) and (b) dominant (nuclear). Given that in many cases most of the starburst activity seem to originate in the nuclear part of the galaxy, we have also considered a third intermediate case $(0.2$ off $+0.8 n u c)$ in which $80 \%$ of the starburst is contained in nucleus and the remaining $20 \%$ is distributed in the rest of the galaxy. Such a scenario is conservative but compatible with all the observations mentioned in Appendix A.

The rates are computed both for core-collapse $\mathrm{SNe}$ only and also by including the type Ia events. The results are summarized in Table 8.

In each case, we have computed the control time (i.e., the amount of time a SN remains visible in our images) for each galaxy in Table 1 by using the average type Ia light curve and, for the core-collapse events, the light curve of SN 1980K and the upper- and lower envelopes in Fig. 2. From the control time we computed the expected number of detected SNe using the galaxy's $B$ and FIR luminosities and using the appropriate limiting magnitude (see discussion in Sect. 4). The control times computed in this way corresponding to the off-nuclear limits are listed in Table 7. Table 8 gives the expected number of detections for the whole sample and the corresponding number of detections.

The uncertainties on the number of expected events are due to (1) the spread of the $\mathrm{SN} K$-band luminosity (see Fig. 2), (2) the uncertainties in the ratio between $B$ luminosity and SN rate, and (3) the spread of the limiting magnitude in our images. The first effect is the dominant one: for example, when using the average light curve (SN 1980K) and the off-nuclear limits, 0.70 core-collapse $\mathrm{SNe}$ are expected from 
Table 7. Control time (days) for each galaxy for core-collapse (cc) and type Ia SNe using the off-nuclear limiting magnitude.

\begin{tabular}{|c|c|c|c|}
\hline Galaxy & $N$ obs. & $\begin{array}{l}\text { CT(cc) } \\
\text { (days) }\end{array}$ & $\begin{array}{l}\text { CT(Ia) } \\
\text { (days) }\end{array}$ \\
\hline NGC 34 & 6 & 457.1 & 332.3 \\
\hline NGC 232 & 3 & 399 & 265.5 \\
\hline $\mathrm{MCG}+12-02-001$ & 4 & 325.5 & 260.1 \\
\hline IC 1623 & 5 & 416.6 & 287.2 \\
\hline UGC 2369 & 5 & 338.1 & 244.4 \\
\hline IRAS0335+15 & 8 & 324.7 & 211.4 \\
\hline MCG-03-12-00 & 4 & 300.6 & 199.2 \\
\hline NGC 1572 & 5 & 467.2 & 343.8 \\
\hline NGC 1614 & 13 & 746.4 & 583.9 \\
\hline IRAS0518-25 & 4 & 265.4 & 184.2 \\
\hline ESO255-IG007 & 6 & 346.8 & 258.4 \\
\hline NGC 2623 & 12 & 575.0 & 459.8 \\
\hline IRAS0857+39 & 5 & 43.9 & 27.8 \\
\hline UGC 4881 & 7 & 181.2 & 121.6 \\
\hline UGC 5101 & 7 & 181.1 & 122.3 \\
\hline MCG+08-18-012 & 2 & 77.1 & 61 \\
\hline IC 563/IC564 & 3 & 101.1 & 70.1 \\
\hline NGC 3110 & 6 & 405.9 & 305.2 \\
\hline IRAS1017+08 & 5 & 184.8 & 145.9 \\
\hline NGC 3256 & 5 & 582.4 & 458.4 \\
\hline IRAS1056+24 & 8 & 161.0 & 112.6 \\
\hline Arp 148 & 4 & 181.0 & 94.8 \\
\hline MCG+00-29-023 & 6 & 325.4 & 248 \\
\hline IC 2810/UGC6436 & 4 & 188.7 & 125.6 \\
\hline NGC 3690 & 9 & 555.1 & 442.7 \\
\hline IRAS1211+03 & 5 & 102.6 & 83.4 \\
\hline ESO507-G070 & 2 & 208.5 & 143 \\
\hline UGC 8335 & 5 & 297.1 & 194.5 \\
\hline UGC 8387 & 4 & 221.3 & 149.5 \\
\hline NGC 5256 & 2 & 62.3 & 53.6 \\
\hline NGC 5257 & 6 & 360.0 & 273.1 \\
\hline Mk 273 & 4 & 131.3 & 77.1 \\
\hline NGC 5331 & 4 & 242.8 & 149.3 \\
\hline Arp 302 & 5 & 215.4 & 115.8 \\
\hline Mk 848 & 3 & 116.4 & 70 \\
\hline IRAS1525+36 & 2 & 0.0 & 0 \\
\hline Arp 220 & 12 & 591.9 & 478.4 \\
\hline NGC 6090 & 4 & 212.1 & 164.7 \\
\hline IRAS16164-0746 & 2 & 195.2 & 115.3 \\
\hline NGC 6240 & 10 & 459.3 & 380.9 \\
\hline IRAS17208-0014 & 5 & 125.6 & 75.8 \\
\hline IC 4687/86 & 3 & 512.1 & 350.4 \\
\hline IRAS1829-34 & 3 & 503.8 & 325.8 \\
\hline NGC 6926 & 4 & 202.6 & 143.2 \\
\hline NGC 7130 & 3 & 436.2 & 313.2 \\
\hline
\end{tabular}

the B luminosity, while 0.90 and 0.46 events are expected when using the light curves corresponding, respectively, to the upper and lower envelope of Fig. 2.

From Table 8 it is apparent that in all cases the number of SNe predicted from the $B$ luminosity, between 0.31 and 0.84 , is much lower than our 4 detections. When the $\mathrm{SN}$ rate is estimated from the FIR luminosity we obtain drastically different results: the expected number of $\mathrm{SNe}$ is higher by a factor of 3-10 than the SNe actually detected (see next section).
Table 8. Number of observed SNe compared with the number of expected events as derived from the $B$ and FIR luminosities. cc indicates core-collapse $\mathrm{SNe}$ only, cc+Ia includes also type Ia SNe.

\begin{tabular}{lr|cc}
\hline \hline & & cc & cc+Ia \\
\hline Detected SNe & off-nuclear & $0.70 \pm 0.22$ & $0.84 \pm 0.23$ \\
\hline \multirow{2}{*}{ Expec. from $L_{B}$} & nuclear & $0.25 \pm 0.22$ & $0.31 \pm 0.23$ \\
& 0.2off + 0.8nuc & $0.34 \pm 0.18$ & $0.49 \pm 0.18$ \\
\hline & off-nuclear & $35 \pm 16$ & $42 \pm 17$ \\
Expec. from $L_{\mathrm{FIR}}$ & nuclear & $10 \pm 8$ & $11 \pm 8$ \\
& 0.2 off + 0.8nuc & $15 \pm 7$ & $18 \pm 7$ \\
\hline
\end{tabular}

The number of the detected SNe can also be used to derive a near-IR $S N$ rate $\left(\mathrm{SN}_{\mathrm{r}}^{\mathrm{NIR}}\right)$, i.e., based on near-IR observations. We consider only the case with $80 \%$ of FIR luminosity coming from the nucleus. The rates were computed by dividing the total number of $\mathrm{SNe}$ by the sum of the luminosity of the galaxies times the control time. By using both the $B$ luminosity and $L_{\text {FIR }}$ we obtain, respectively:

$\mathrm{SN}_{\mathrm{r}}^{\mathrm{NIR}}=7.6 \pm 3.8 \pm 2.8 \mathrm{SNu}(B)$
$\mathrm{SN}_{\mathrm{r}}^{\mathrm{NIR}}=0.53 \pm 0.27 \pm 0.21 \mathrm{SNuIR}$.

The two errors terms in each equation are due to the statistics of the number of detections and the uncertainties in the computation of the control time, respectively. It should be noted that the first one (statistics) is the dominant term due to the small number of detections, and that the second one (control time) is not a standard " $1 \sigma$ " $68 \%$ value but rather a total $100 \%$ uncertainty.

\section{Comparison between expected and detected $\mathrm{SN}$ rates}

The first conclusion is that NIR searches for SN in starburst galaxies are, as expected, more efficient than similar searches at optical wavelengths since at least some of the SNe occurs in dusty environments. Two SNe were in fact only detected in the NIR, and Maiolino et al. (2002) demonstrated that at least SN $2001 \mathrm{db}$ was too absorbed $\left(A_{V} \sim 5.6 \mathrm{mag}\right)$ to be detected during routine optical $\mathrm{SN}$ surveys even at its maximum. This is also the case for the type Ia SN 2002cv discovered in the NIR by Di Paola et al. (2002) and for which Meikle \& Mattila (2002) derived an extinction in excess of $A_{V}=6 \mathrm{mag}$.

The second conclusion is that we are detecting more $\mathrm{SNe}$ than expected from the $B$ luminosity, both considering or neglecting the contribution from type Ia SNe. The hypothesis that the detected number of core-collapse $\mathrm{SNe}$ (3) is still compatible with the expectations $(0.34)$ can be rejected at more than the $99.5 \%$ confidence level. A very similar result is obtained if we include the type Ia SNe ( 0.49 predicted vs. 4 observed).

This high value for the observed $\mathrm{SN}$ rate reflects the higher extinction affecting the $B$ light (which is the normalizing factor of $\mathrm{SNu}$ ) of the galaxies in our sample, and the higher SN detection efficiency of the NIR observations with respect to the optical.

This result is not only due to obscuration, as 2 of the 4 detected SNe were also observed in the optical. For this reason 
the low success rate of previous NIR surveys for $\mathrm{SNe}$ is probably due to modest star forming activity of the monitored galaxy sample: for this work we used galaxies with higher rates of star formation which translates into a higher expected $\mathrm{SN}$ rate.

The third conclusion is that the majority of SNe expected from the FIR luminosity are still missing, i.e., we have detected only about $10-30 \%$ of the expected SNe. This small number of detections can be explained in several ways:

1. Most of the SNe are so embedded into the dust that their luminosity is vastly reduced even at near-IR wavelengths. The average extinction $A_{V}$ needed to reduce the expected number of $15 \mathrm{SNe}$ to the observed number of 4 is $A_{V}=$ $31 \mathrm{mag}$, or $A_{V}>26$ (corresponding to $8 \mathrm{SNe}$ ) with a formal confidence level of $90 \%$. These values for the extinction are similar to those obtained by Genzel et al. (1998) from mid-infrared spectroscopy of several luminous galaxies, and by Mattila \& Meikle (2001) for M82 by assuming a gas-to-dust ratio similar to the Milky Way. In this case, a NIR search for SN can detect only a fraction of the total $\mathrm{SN}$ rate, and a radio survey should also be planned.

2. If $100 \%$ of the FIR flux comes from the central arcsec, only 11 events are expected because of the residuals present in the nuclear region even after applying a PSF matching algorithm. When considering also the uncertainties in the predictions, dominated by the dispersion in luminosity of the $\mathrm{SNe}$, the number of expected $\mathrm{SNe}$ is compatible with the observed one without the need of a large additional extinction. The presence of a large number of $\mathrm{SNe}$ in the central arcsec of the starburst galaxies can be tested by nearIR monitoring from space: NICMOS on HST can provide images with higher resolution $\left(\simeq 0.2^{\prime \prime}\right)$ and the more stable PSF to considerably reduce the central residuals and reveal the nuclear SNe. Early results on such a project based on 4 galaxies will be presented in a forthcoming paper (Cresci et al. 2003, in preparation).

3. Another possibility is the presence of AGNs dominating the FIR flux of most of the galaxies: in this case the FIR flux would not be related to the $\mathrm{SN}$ rate. There is evidence that the AGN does not dominate the energetics of the galaxies with $L_{\mathrm{FIR}}<2 \times 10^{12} L_{\odot}$ (e.g., Sanders \& Mirabel 1996). Nevertheless it is sometimes difficult to exclude the presence of an AGN completely enshrouded by dust and therefore elusive in the optical/IR or even in the X-rays (Marconi et al. 2000; Maiolino et al. 1998).

4. Equation (4) may overestimate the SN rate for a given FIR luminosity. Many of the points in Fig. 3 and in particular that for the most luminous galaxy NGC 7496 are, directly or indirectly, derived from radio observations and make use of the empirical relations by Condon \& Yin (1990). In turn, this is based on radio observations of the Milky Way and on the estimate of its SN rate (Tammann 1982). If the SN radio luminosity has a strong dependence on environment or if the estimates of the Galactic SN rate are subject to strong incompleteness, the coefficient in Eq. (4) could be inadequate. We believe that this is not a likely possibility as there is a robust concordance among several different methods and this relation has been stable through the years (see, for example, Rieke et al. 1980).

5. Part of the difference could also be due to a different IMF in the starburst regions with respect to more quiescent galaxies. Such an effect, studied in M 82 by Rieke et al. (1993), could change the relation between luminosity and $\mathrm{SN}$ rate. If present, it is not likely to be a dominant effect as both the FIR luminosity and the core-collapse SNe derive from massive stars.

6. An environment dependence could also be present in the SN luminosity: as an example, $\mathrm{SN}$ in dense environments are expected to show a flatter light curve because of the presence of a light echo (e.g., Roscherr \& Schaefer 2000). This has some consequences on the expected number of SNe: events showing the "ordinary" decline rate can be detected even if observed only after the maximum light, while the detection of "slow" events need the comparison of images taken before and after the explosion. In the former case the total useful time contains also one control time before the first image, in the latter case only the time between the images should be considered, and the control time is reduced by about $25 \%$. Therefore this effect could be present but is not strong enough to explain the reduced number of detections.

\section{Summary and conclusions}

We have monitored 46 infrared luminous galaxies at nearinfrared wavelengths to look for obscured $\mathrm{SNe}$, and detected $4 \mathrm{SNe}, 3$ core-collapse and 1 type Ia events. The analysis of this data set has allowed us to derive for the first time the infrared $\mathrm{SN}$ rate in starburst galaxies.

To apply the control-time technique, we have collected more than 200 observations of 21 core-collapse SNe to derive their average, maximum and minimum $K$-band luminosities. We have also extended the relation by Mattila \& Meikle (2001) between SN rate and FIR luminosity toward objects with higher SFR in order to cover the luminosity range of the galaxies in our sample. This relation remains linear, i.e., the $\mathrm{SN}$ rate is proportional to the FIR luminosity, pointing toward a substantial uniformity in this property between quiescent and active galaxies.

We have computed the rate of type Ia $\mathrm{SN}$ as a function of the stellar mass of the galaxy, finding a clear evidence of its increase toward galaxies with higher activity of star formation. This is an other indication that a fraction of type Ia SNe is probably be associated with relatively young stellar populations.

We estimated the expected SN rate from the optical $(B)$ and FIR luminosities of the sample of galaxies and compared those predictions to the number of detections. The SN rate predicted from the $B$-band luminosity is several times below the observed rate. This is likely to be explained by a number of reasons, the most important of which is the effect of dust obscuration on the optical luminosity of the galaxies. On the contrary, a factor of 3-10 more SNe than observed are predicted by the FIR luminosity. Among various possibilities, this can be explained by the presence of dust extinction of at least $A_{V}=30$. This result prompts to the revision of the local $\mathrm{SN}$ rate obtained in the 
past, as optical monitoring campaigns have probably missed a significant fraction of SNe.

Acknowledgements. We are grateful to S. Mattila and P. Meikle for useful discussions and to Marcia and George Rieke for the use of the AZ61 near-infrared camera.

\section{Appendix A: The spatial dimension of the starburst activity}

The spatial resolution at $100 \mu \mathrm{m}$ is too low to image the spatial distribution of the starburst activity: the highest resolution achieved of this type of galaxies is about $23^{\prime \prime}$ by Zink et al. (2000), much larger that the scale of about $2^{\prime \prime}$ of importance here. Even at this coarse resolution, these authors were able to clearly resolve 6 of their 22 galaxies, and obtain "some evidence for extension" in 7 more objects: as a result about two third of this incomplete sample have $100 \mu \mathrm{m}$ dimension above $20^{\prime \prime}$.

Indirect evidence, but on a smaller scale, can be found by considering the images at mid-infrared wavelengths. The tight correlation existing between the emission at $100 \mu \mathrm{m}$ and at $15 \mu \mathrm{m}$ for galaxies over 4 orders of magnitudes of luminosity (Elbaz et al. 2002) implies that the mid-infrared should trace the $100 \mu \mathrm{m}$ emission (unless an AGN is present). High resolution mid-infrared images of luminous infrared galaxies were obtained by Dale et al. (2000) using ISO. Most of the galaxies show emission coming from several kpc of size, corresponding to several (2-20) arcsec in our galaxies. A possible correlation is also seen between $L_{\mathrm{FIR}}$ and the size of the region emitting $80 \%$ of the $15 \mu \mathrm{m}$ emission (see Fig. A.1) for the galaxies with $L_{\mathrm{FIR}}>10^{10} L_{\odot}$. According to this relation, no starburst region in the galaxies in our sample should have dimensions below 4 ".

Bushouse et al. (1998) studied the relative distributions of several indicators of star formation, such as $\mathrm{H} \alpha$, mid-infrared, far-infrared and radio continuum, and found a good correlation between these indicators in the central regions of a few nearby galaxies. At $10 \mu \mathrm{m}$, the emitting source is usually not resolved at their resolution of about $5^{\prime \prime}$, indicating that most of the starburst is concentrated in the nucleus. The upper limits to the size of the far-infrared region are generally compatible with those from the mid-infrared. In one case, NGC $660\left(\log \left(L_{\mathrm{FIR}} / L_{\odot}\right)=\right.$ $10.38)$, the infrared emission is resolved both in the mid- and in the far-infrared: at $10 \mu \mathrm{m}$ Bushhouse et al. (1998) found a size of about 320 pc, while Smith \& Harvey (1996) obtained about $2 \mathrm{kpc}$ at $100 \mu \mathrm{m}$. At least in this case the far-infrared emission seems more extended than at shorter wavelengths.

Soifer et al. (2001) imaged a small number of highluminosity galaxies with Keck at $12.5 \mu$ m with a field-of-view of $17^{\prime \prime} \times 17^{\prime \prime}$. They generally find that most of the emission comes from the central 2-3", much more compact regions than found in the sample by Dale et al. (2000). This result is somewhat expected because of the small field-of-view, but suggests once more that the nuclear star formation contributes most of the mid- and far-IR.

In conclusions, current data don't put tight constraints of the dimension of the starburst activity and therefore it is not

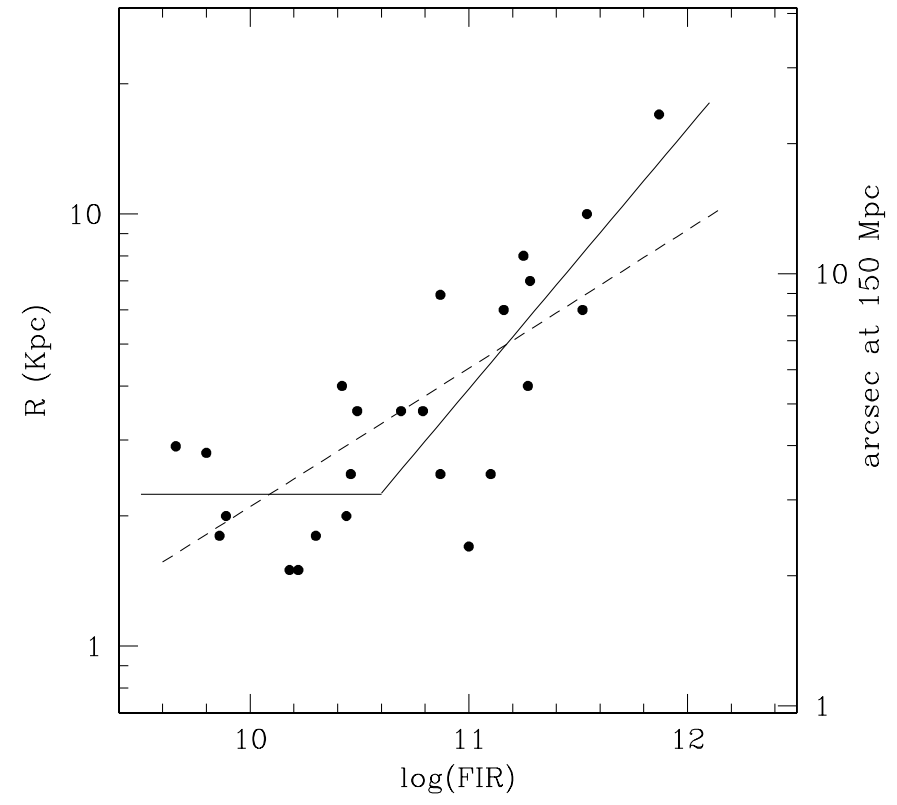

Fig. A.1. Size of the region emitting $80 \%$ of the luminosity at $15 \mu \mathrm{m}$ vs. the FIR luminosity. The data points are the galaxies present in both the Dale et al. (2000) and Soifer et al. (1987) catalogs. The dashed line shows the linear fit to all the points, while the solid lines are the linear fit for $L_{\mathrm{FIR}}>4 \times 10^{10} L_{\odot}$ and the average below this limit.

possible to estimate the amount of FIR flux originating the in the central 2 arcsec. For this reason we have computed the SN rate in the two limiting cases that none and all the FIR flux comes from the nuclear region. Given that most galaxies seem to be dominated by nuclear starburst we have also computed the SN rate by assuming the $80 \%$ of the FIR flux originates from the nucleus and $20 \%$ from the rest of the galaxy.

\section{References}

Alard, C., \& Lupton, R. H. 1998, ApJ, 503, 325

Alard, C. 2000, A\&AS, 144, 363

Arnett, W. D., Bahcall, J. N., Kirshner, R. P., \& Woosley, S. E. 1989, ARA\&A, 27, 629

Baffa, C., Comoretto, G., Gennari, S., et al. 2001, A\&A, 378, 722

Bell, E. F., \& de Jong, R. S., ApJ, 550, 212

Bertin, E., \& Arnouts, S. 1996, A\&AS, 117, 393

Bouchet, P., Moneti, A., Slezak, E., Le Bertre, T., \& Manfroid, J. 1989, A\&AS, 80, 379

Branch, D., \& van den Bergh, S. 1993, AJ, 105, 2231

Bregman, J. D., Temi, P., \& Rank, D. 2000, A\&A, 355, 525

Bressan, A., Della Valle, M., \& Marziani, P. 2002, MNRAS, 331, L25

Brinchmann, J., \& Ellis, R. S. 2000, ApJ, 536, L77

van Buren, D., \& Norman, C. A. 1989, ApJ, 336, L67

van Buren, D., \& Greenhouse, M. A. 1994, ApJ, 431, 640

van Buren, D., Jarrett, T., Terebey, S., et al. 1994, IAUC, 5960, 2

Bushouse, H., Telesco, C. M., \& Werner, M. W. 1998, AJ, 115, 938

Calamai, G., Gavazzi, G., Randone, I., \& Tofani, G. 1993, IAU Circ., 5741,1

Cappellaro, E., \& Turatto, M. 2001, in The influence of binaries on stellar population studies, ed. D. Vanbeveren

Cappellaro, E., Evans, R., \& Turatto, M. 1999, A\&A, 351, 459

Clocchiatti, A., Wheeler, J. C., Phillips, M. M., et al. 1997, ApJ, 483, 675 
Condon, J. J., \& Yin, Q. F. 1990, ApJ, 357, 97

Corbett, E. A., Norris, R. P., Heisler, C. A., et al. 2002, ApJ, 564, 650

Cram, L., Hopkinns, A., Mobasher, B., \& Rowan-Robinson, M. 1998, ApJ, 507, 155

Cresci, G., Mannucci, F., Maiolino, R., Della Valle, M., \& Ghinassi, F. 2002, IAU Circ., 7784

Dale, D. A., Silbermann, N. A., Helou, G., et al. 2000, AJ, 120, 583

Dahlén, T., \& Fransson, C. 1999, A\&A, 350, 349

Della Valle, M., \& Livio, M. 1994, A\&A, 287, 403

Della Valle, M., \& Panagia, N. 1992, AJ, 104, 696

Della Valle, M., \& Panagia, N. 2003, ApJL, in press

Di Carlo, E., Massi, F., Valentini, G., et al. 2002, A\&A, L393, 1

Dwek, A'Hearn, M. F., Becklin, E., et al. 1983, ApJ, 274, 168

Elbaz, D., Cesarsky, C. J., Chanial, P., et al. 2002, A\&A, 384, 848

Elias, J. H., Matthews, K., Neugebauer, G., \& Persson, S. E. 1985, ApJ, 296, 379

Fassia, A., Meikle, W. P. S., Chugai, N., et al. 2001, MNRAS, 325, 907

Fassia, A., Meikle, W. P. S., Vacca, W. D., et al. 2000, MNRAS, 318, 1093

Filippenko, A. V., \& Garnavich, P. 1999, IAU Circ., 7328

Fioc, M., \& Rocca-Volmerange, B. 1999, A\&A, 351, 869

Genzel, R., Lutz, D., Sturm, E., et al. 1998, ApJ, 498, 579

Gerardy, C. L., Fesen, R. A., Nomoto, K., et al. 2002, ApJ, 575, 1007

Graham, J. R. 1985, Ph.D. Thesis, Imperial College of Science and Technology, London, UK

Grossan, B., Spillar, E., Tripp, R., et al. 1999, AJ, 118, 705

Hackenberg, W., Eckart, A., Davies, R. I., et al. 2000, A\&A, 363, 41

Hamuy, M., Trager, S. C., Pinto, P. A., et al. 2000, AJ, 120, 1479

Hamuy, M., Pinto, P. A., Maza, J., et al. 2001, ApJ, 558, 615

Helou, G., Khan, I. R., Malek, L., \& Boehmer, L. 1988, ApJS, 68, 151

Hunt, L. K., Mannucci, F., Testi, L., et al. 1998, AJ, 115, 2594

Jha, S., \& Brown, W. 2000, IAU Circ., 7394, 2

Kidger, M., Mahoney T., \& Selby, M. 1993, IAU Circ., 5795, 2

Kim, D.-C., \& Sanders, D. B. 1998, ApJS, 119, 41

Lawrence, G. F., Paulson, A., Mason, C., Butenhoff, C., \& Gehrz, R. D. 1993, IAU Circ., 5844, 3

Li, W. D. 1999, IAUC, 7319, 1

Lidman, C., Cuby, J.-G., \& Vanzi, L. 2000, SOFI User's Manual, ESO

Lisi, F., Baffa, C., \& Hunt, L. K. 1993, SPIE, 1946, 594

Lorionov, V., Arkharov, A., Caratti, A, Di Paola, A., \& Dolci, M. 2002, IAU Circ., 7901

Madau, P., Della Valle, M., \& Panagia, N. 1998, MNRAS, 297, L17

Maiolino, R., Salvati, M., Bassani, L., et al. 1998, A\&A, 338, 781

Maiolino, R., Della Valle, M., Vanzi, L., \& Mannucci, F. 2001, IAU Circ., 7661, 2

Maiolino, R., Vanzi, L., Mannucci, F., et al. 2002, A\&A, 389, 84

Mannucci, F., \& Beckwith, S. V. W. 1995, ApJ, 442, 569

Marconi, A., Oliva, E., \& van der Werf, P. P. 2000, A\&A, 357, 24

Matthews, K., Neugebauer, G., Armus, L., \& Soifer, B. T. 2002, AJ, 123,753

Mattila, S., \& Meikle, W. P. S. 2001, MNRAS, 324, 325
Mattila, S., \& Meikle, W. P. S. 2002, IAU Circ., 7820, 1

Mattila, S., Meikle, P., Walton, N., et al. 2002, IAU Circ., 7865

Meikle, W. P. S. 2000, MNRAS, 314, 782

Meikle, W. P. S., \& Mattila, S. 2002, IAU Circ., 7911, 1

Motohara, K., Nomoto, K., Gerardy, C., Fesen, R., \& Henry, P. 2002, IAU Circ., 7884, 1

Muller, A., Pizarro, O., Westerlund, B., \& Dennefeld, M. 1982, IAU Circ., 3739, 1

Navasardyan, H., Petrosian, A. R., Turatto, M., Cappellaro, E., \& Boulesteix, J. 2001, MNRAS, 328, 1181

Neff, S. G., \& Ulvestad, J. S. 2000, AJ, 120, 670

Odewahn, S., \& Bailey, J. 1993, IAU Circ., 5762, 1

Patat, F., Cappellaro, E., Danziger, J., et al. 2001, ApJ, 555, 900

Persson, S. E., Murphy, D. C., Krzeminski, W., Roth, M., \& Rieke, M. J. 1998, AJ, 116, 2476

Richmond, M. W., Filippenko, A. V., \& Galisky, J. 1998, PASP, 110, 553

Rieke, G. H., Loken, K., Rieke, M. J., \& Tamblyn, P. 1993, ApJ, 412, 99

Rieke, G. H., Lebofsky, M. J., Thompson, R. I., Low, F. J., \& Tokunaga, A. T. 1980, ApJ, 238, 24

Romanishin, W. 1993, IAU Circ., 5773, 1

Roscherr, B., \& Schaefer, B. 2000, ApJ, 535, 415

Sanders, D. B., \& Mirabel, I. F. 1996, ARA\&A, 34, 749

Sanders, D. B., Egami, E., Lipari, S., Mirabel, I. F., \& Soifer, B. T. 1995, AJ, 110, 1993

Sato, Y., \& Li, W. D. 2000, IAUC, 7392, 1

Schaerer, D. 1999, in Building the Galaxies: from the Primordial Universe to the Present, XIXth Moriond astrophysics meeting, ed. F. Hammer, T. X. Thuan, V. Cayatte, B., Guiderdoni, \& J. T. Thanh Van (Gif-sur-Yvette: Éditions Frontières)

Schlegel, E. M. 1990, 244, 269

Smith, J. A. 1993, IAU Circ., 5780, 1

Smith, B. J., \& Harvey, P. M. 1996, ApJ, 468, 139

Smith, D. A., Herter, T., Haynes, M. P., \& Neff, S. 1999, ApJ, 510, 669

Soifer, B. T., Sanders, D. B., Madore, B. F., et al. 1987, ApJ, 320, 238

Soifer, B. T., Neugebauer, G., Matthews, K., et al. 2001, ApJ, 559, 201

Sullivan, M., Ellis, R., Nuget, P., Smail, I., \& Madau, P. 2000, MNRAS, 319, 549

Tammann, G. A. 1982, in Supernovae: A Survey of Current Research, ed. M. J. Rees, \& R. J. Stonehan (Dordrecht: Reidel), 371

Thean, A., Pedlar, A., Kukula, M. J., Baum, S. A., \& O’Dea, C. P. 2001, MNRAS, 325, 737

Vink, J. S., de Koter, A., \& Lamers, H. J. G. L. M. 2001, A\&A, 369, 574

Wilson, A. S., Helfer, T. T., Haniff, C. A., \& Ward, M. J. 1991, ApJ, 381, 79

Yoshii, Y., Minezaki, T., Aoki, T., et al. 2002, IAU Circ., 7826, 1

Zink, E. C., Lester, D. F., Doppmann, G., \& Harvey, P. 2000, ApJS, 131, 413

Zwicky, F. 1938, ApJ, 88, 529 